\title{
Precise Point Positioning with Kepler
}

\author{
Patrick Henkel \\ Institute of Communications and Navigation \\ German Aerospace Center (DLR) \\ Oberpfaffenhofen, Germany \\ patrick.henkel@dlr.de
}

\begin{abstract}
Precise Point Positioning (PPP) enables an absolute positioning with centimeter-level accuracy without the need of raw measurements from a reference station. However, today's GPS L1/ L2-based PPP solutions typically need more than 30 minutes to converge.

In this paper, we present a PPP solution with a much faster convergence using the proposed next-generation GNSS Kepler. We exploit the high accuracy of the satellite position, clock offset and bias estimates enabled by highly accurate optical intersatellite range measurements. We additionally exploit the low noise level of the E1, E5 and E6 pseudorange measurements based on wideband signals as provided already by Galileo. Our PPP solution determines the receiver position and clock offset, tropospheric and ionospheric zenith delays, and pseudorange multipath errors without the need of any prior information. We show that the PPP convergence time can be reduced from more than 30 minutes to less than 5 minutes with Kepler.
\end{abstract}

Index Terms-Precise Point Positioning, Satellite Navigation, Kalman filter, Next-Generation GNSS, Kepler.

\section{INTRODUCTION}

PPP was first described in [1]. It requires precise satellite position, clock offset and bias estimates. These estimates are derived today in global network solutions with typically more than 100 ground stations. The next generation GNSS Kepler proposed by [2] uses highly accurate optical intersatellite links, which enables a very accurate estimation of satellite position, clock offset and biases without the need of an extensive ground infrastructure. The L-band signals of Kepler are equal to those of Galileo, i.e. the wideband signals on E5 and E6 provide pseudoranges with centimetre accuracy.

\section{Measurement Model}

This section begins with a brief description of the traditional model for absolute carrier phase and pseudorange measurements for Precise Point Positioning (PPP). Subsequently, we adapt our model to the proposed next generation GNSS Kepler, i.e. we exploit the benefits achieved through optical inter-satellite links.

\section{A. Standard measurement model}

The absolute carrier phase measurement tracked by user $u$ from satellite $k=\{1, \ldots, K\}$ on frequency $m=\{1, \ldots, M\}$

This work was supported by the project ADVANTAGE, funded in part by the Helmholtz association (Grant ZT-0007, Jan. 2017). at time $t_{n}$ is modeled according to [3] as:

$$
\begin{aligned}
\lambda_{m} & \varphi_{u, m}^{k}\left(t_{n}\right) \\
= & \left\|\vec{x}_{u}\left(t_{n}\right)+\Delta \vec{x}_{\mathrm{ET}}-\hat{\vec{x}}^{k}\left(t_{n}-\Delta t_{n}\right)-\Delta \vec{x}^{k}\left(t_{n}\right)\right\| \\
& +c\left(\delta \tau_{u}\left(t_{n}\right)-\delta \hat{\tau}^{k}\left(t_{n}-\Delta t_{n}\right)-\Delta \delta \tau^{k}\left(t_{n}\right)\right) \\
& +m_{\mathrm{T}}\left(\theta_{u}^{k}\left(t_{n}\right)\right) \cdot T_{\mathrm{z}, u}\left(t_{n}\right)-q_{1 m}^{2} I_{u, 1}^{k}\left(t_{n}\right)+\lambda_{m} N_{u, m}^{k} \\
& +\Delta \varphi_{\mathrm{PW}, u}^{k}\left(t_{n}\right)+\Delta \varphi_{\mathrm{PCO}, u}^{k}+\Delta \varphi_{\mathrm{PCV}, u}^{k}\left(t_{n}\right) \\
& +\beta_{u, m}-\beta_{m}^{k}+\Delta \varphi_{\mathrm{MP}, u, m}^{k}\left(t_{n}\right)+\varepsilon_{u, m}^{k}\left(t_{n}\right),
\end{aligned}
$$

with the following notations:

$\begin{array}{ll}\lambda_{m} & \text { wavelength } \\ \varphi_{u, m}^{k} & \text { carrier phase measurement in unit of cycles } \\ \vec{x}_{u} & \text { receiver position } \\ \Delta \vec{x}_{\mathrm{ET}} & \text { Earth tide correction } \\ \hat{\vec{x}}^{k} & \text { est. sat. position based on broadcast orbits } \\ \Delta t_{n} & \text { propagation time from sat. to receiver } \\ \Delta \vec{x}^{k} & \text { error of satellite position estimate } \\ c & \text { speed of light in vacuum } \\ \delta \tau_{u} & \text { clock offset of the receiver } \\ \delta \hat{\tau}^{k} & \text { est. sat. clock offset based on broadcast clocks } \\ \Delta \delta \tau^{k} & \text { error of sat. clock offset estimate } \\ m_{\mathrm{T}} & \text { tropospheric mapping function } \\ \theta_{u}^{k} & \text { satellite elevation angle at receiver } \\ T_{\mathrm{Z}, u} & \text { tropospheric zenith delay at receiver } \\ q_{1 m}^{2} & \text { squared ratio between frequencies } f_{1} \text { and } f_{m} \\ I_{u, 1}^{k} & \text { ionospheric slant delay on } f_{1} \\ N_{u, m}^{k} & \text { carrier phase integer ambiguity } \\ \Delta \varphi_{\mathrm{PW}, u}^{k} & \text { phase wind-up } \\ \Delta \varphi_{\mathrm{PCO}, u}^{k} & \text { phase center offsets of rec. and sat. ant. } \\ \Delta \varphi_{\mathrm{PCV}, u}^{k} & \text { phase center variat. of rec. and sat. ant. } \\ \beta_{u, m} & \text { receiver phase bias } \\ \beta_{m}^{k} & \text { satellite phase bias } \\ \Delta \varphi_{\mathrm{MP}, u, m}^{k} & \text { carrier phase multipath error } \\ \varepsilon_{u, m}^{k} & \text { phase noise } \\ \mathrm{Th} & \end{array}$

The pseudorange measurement as provided by the Delay Locked Loop (DLL) is modeled similarly as:

$$
\begin{aligned}
& \rho_{u, m}^{k}\left(t_{n}\right) \\
&=\left\|\vec{x}_{u}\left(t_{n}\right)+\Delta \vec{x}_{\mathrm{ET}}-\hat{\vec{x}}^{k}\left(t_{n}-\Delta t_{n}\right)-\Delta \vec{x}^{k}\left(t_{n}\right)\right\| \\
&+c\left(\delta \tau_{u}\left(t_{n}\right)-\delta \hat{\tau}^{k}\left(t_{n}-\Delta t_{n}\right)-\Delta \delta \tau^{k}\left(t_{n}\right)\right) \\
&+m_{\mathrm{T}}\left(\theta_{u}^{k}\left(t_{n}\right)\right) \cdot T_{\mathrm{z}, u}\left(t_{n}\right)+q_{1 m}^{2} I_{u, 1}^{k}\left(t_{n}\right) \\
&+b_{u, m}-b_{m}^{k}+\Delta \rho_{\mathrm{MP}, u, m}^{k}\left(t_{n}\right)+\eta_{u, m}^{k}\left(t_{n}\right),
\end{aligned}
$$


with the following additional parameters:

$$
\begin{array}{ll}
b_{u, m} & \text { receiver code bias } \\
b_{m}^{k} & \text { satellite code bias } \\
\Delta \rho_{\mathrm{MP}, u, m}^{k} & \text { pseudorange multipath error } \\
\eta_{u, m}^{k} & \text { pseudorange noise }
\end{array}
$$

\section{B. Adaptions for Kepler}

Kepler has been proposed by [2] as next-generation GNSS. It uses optical inter-satellite range measurements, that are several orders of magnitude more accurate than current L-band measurements and that are not affected by atmospheric errors. Therefore, the satellite orbits and clocks can be determined with sub-centimeter level accuracy in real-time. The residual satellite position error $\Delta \vec{x}^{k}$ and satellite clock error $\Delta \delta \tau^{k}$ can be neglected in Eq. (1) and (2) for PPP with Kepler. This also implies that the satellite phase biases $\beta_{m}^{k}$ and pseudorange biases $b_{m}^{k}$ can be estimated very accurately.

\section{CORRECTION AND RE-PARAMETRIZATION OF MEASUREMENTS}

In this section, the absolute phase and pseudorange measurements are corrected for the broadcast orbits and clocks and other parameters known through accurate models. Subsequently, we re-parameterize the corrected measurements to obtain a full-rank system of equations, which allows the joint estimation of the absolute receiver position and clock offset, tropospheric zenith delay, ionospheric slant delays, carrier phase ambiguities and pseudorange multipath errors.

\section{A. Pre-correction of measurements}

The absolute carrier phase measurement of Eq. (1) is precorrected for the known Earth tides, satellite position and clock estimates, phase-wind up, phase center offset and variation, and satellite phase biases, i.e.

$$
\begin{aligned}
\lambda_{m} \tilde{\varphi}_{u, m}^{k}\left(t_{n}\right):= & \lambda_{m} \varphi_{u, m}^{k}\left(t_{n}\right) \\
& -\left(\vec{e}_{u}^{k}\left(t_{n}\right)\right)^{\mathrm{T}}\left(\Delta \vec{x}_{\mathrm{ET}}-\hat{\vec{x}}^{k}\left(t_{n}-\Delta t_{n}\right)\right) \\
& +c \delta \hat{\tau}^{k}\left(t_{n}-\Delta t_{n}\right)-\Delta \varphi_{\mathrm{PW}, u}^{k}\left(t_{n}\right) \\
& -\Delta \varphi_{\mathrm{PCO}, u}^{k}-\Delta \varphi_{\mathrm{PCV}, u}^{k}\left(t_{n}\right)+\hat{\beta}_{m}^{k} \\
= & \left(\vec{e}_{u}^{k}\left(t_{n}\right)\right)^{\mathrm{T}} \vec{x}_{u}\left(t_{n}\right)+c \delta \tau_{u}\left(t_{n}\right) \\
& +m_{\mathrm{T}}\left(\theta_{u}\left(t_{n}\right)\right) \cdot T_{\mathrm{z}, u}\left(t_{n}\right)-q_{1 m}^{2} I_{u, 1}^{k}\left(t_{n}\right) \\
& +\lambda_{m} N_{u, m}^{k}+\beta_{u, m}+\varepsilon_{u, m}^{k}\left(t_{n}\right),
\end{aligned}
$$

with the normalized direction vector $\vec{e}_{u}^{k}=\left(\vec{x}_{u}-\vec{x}^{k}\right) / \| \vec{x}_{u}-$ $\vec{x}^{k} \|$ pointing from satellite $k$ to receiver $u$. The pseudorange measurements are corrected similarly as:

$$
\begin{aligned}
\tilde{\rho}_{u, m}^{k}\left(t_{n}\right):= & \rho_{u, m}^{k}\left(t_{n}\right) \\
& -\left(\vec{e}_{u}^{k}\left(t_{n}\right)\right)^{\mathrm{T}}\left(\Delta \vec{x}_{\mathrm{ET}}-\hat{\vec{x}}^{k}\left(t_{n}-\Delta t_{n}\right)\right) \\
& +c \delta \hat{\tau}^{k}\left(t_{n}-\Delta t_{n}\right)+\hat{b}_{m}^{k} \\
= & \left(\vec{e}_{u}^{k}\left(t_{n}\right)\right)^{\mathrm{T}} \vec{x}_{u}\left(t_{n}\right)+c \delta \tau_{u}\left(t_{n}\right) \\
& +m_{\mathrm{T}}\left(\theta_{u}\left(t_{n}\right)\right) \cdot T_{\mathrm{z}, u}\left(t_{n}\right)+q_{1 m}^{2} I_{u, 1}^{k}\left(t_{n}\right) \\
& +\Delta \rho_{\mathrm{MP}, u, m}^{k}\left(t_{n}\right)+b_{u, m}+\eta_{u, m}^{k}\left(t_{n}\right) .
\end{aligned}
$$

The unknowns on the right side are stacked in a state vector. It is given at epoch $n$ by

$$
\boldsymbol{x}_{n}=\left(\begin{array}{c}
\vec{x}_{u}\left(t_{n}\right) \\
c \delta \tau_{u}\left(t_{n}\right) \\
T_{\mathrm{z}, u}\left(t_{n}\right) \\
\boldsymbol{I}_{u, 1}\left(t_{n}\right) \\
\boldsymbol{N}_{u} \\
\Delta \boldsymbol{\rho}_{\mathrm{MP}, u}\left(t_{n}\right)
\end{array}\right)
$$

with

$$
\begin{aligned}
\boldsymbol{I}_{u, 1} & =\left(I_{u, 1}^{1}, \ldots, I_{u, 1}^{K}\right)^{\mathrm{T}} \\
\boldsymbol{N}_{u} & =\left(\boldsymbol{N}_{u, 1}^{\mathrm{T}}, \ldots, \boldsymbol{N}_{u, M}^{\mathrm{T}}\right)^{\mathrm{T}} \\
\boldsymbol{N}_{u, m} & =\left(N_{u, m}^{1}, \ldots, N_{u, m}^{K}\right)^{\mathrm{T}} \\
\Delta \boldsymbol{\rho}_{\mathrm{MP}, u} & =\left(\Delta \boldsymbol{\rho}_{\mathrm{MP}, u, 1}^{\mathrm{T}}, \ldots, \Delta \boldsymbol{\rho}_{\mathrm{MP}, u, M}^{\mathrm{T}}\right)^{\mathrm{T}} \\
\Delta \boldsymbol{\rho}_{\mathrm{MP}, u, m} & =\left(\Delta \rho_{\mathrm{MP}, u, m}^{1}, \ldots, \Delta \rho_{\mathrm{MP}, u, m}^{K}\right)^{\mathrm{T}} .
\end{aligned}
$$

\section{B. Parameter mapping to overcome rank-defect}

The system of Eq. (3) - (6) is under-determined, i.e. it is not possible to separate the receiver clock offset from all ambiguities and pseudorange multipath errors. Therefore, we choose one satellite as reference satellite (being indicated by the upper index ref) and map the respective ambiguity $N_{u, m}^{\mathrm{ref}}$ to the receiver clock offset. The receiver phase bias is also mapped to the receiver clock, and the receiver code bias is mapped to the pseudorange multipath errors. The obtained parameter mapping is given by:

$$
\begin{aligned}
c \delta \tilde{\tau}_{u, m} & :=c \delta \tau_{u}+\beta_{u, m}+\lambda_{m} N_{u, m}^{\mathrm{ref}} \\
\Delta N_{u, m}^{k} & :=N_{u, m}^{k}-N_{u, m}^{\mathrm{ref}} \\
\Delta \tilde{\rho}_{\mathrm{MP}, u, m}^{k} & :=\Delta \rho_{\mathrm{MP}, u, m}^{k}-\lambda_{m} N_{u, m}^{\mathrm{ref}}+b_{u, m}-\beta_{u, m} .
\end{aligned}
$$

The number of multipath parameters is kept equal by this mapping, but the total number of clock offset and ambiguity parameters is reduced from $1+M K$ to $M K$.

The pre-corrected phase measurements of Eq. (3) are expressed in terms of the reduced parameter set of Eq. (7):

$$
\begin{aligned}
\lambda_{m} \tilde{\varphi}_{u, m}^{k}\left(t_{n}\right)= & \left(\vec{e}_{u}^{k}\left(t_{n}\right)\right)^{\mathrm{T}} \vec{x}_{u}\left(t_{n}\right)+c \delta \tilde{\tau}_{u, m}\left(t_{n}\right) \\
& +m_{\mathrm{T}}\left(\theta_{u}\left(t_{n}\right)\right) \cdot T_{\mathrm{z}, u}\left(t_{n}\right)-q_{1 m}^{2} I_{u, 1}^{k}\left(t_{n}\right) \\
& +\lambda_{m} \Delta N_{u, m}^{k}+\varepsilon_{u, m}^{k}\left(t_{n}\right),
\end{aligned}
$$

Similarly, the pre-corrected pseudorange measurements of Eq. (4) are re-parameterized as:

$$
\begin{aligned}
\tilde{\rho}_{u, m}^{k}\left(t_{n}\right)= & \left(\vec{e}_{u}^{k}\left(t_{n}\right)\right)^{\mathrm{T}} \vec{x}_{u}\left(t_{n}\right)+c \delta \tilde{\tau}_{u, m}\left(t_{n}\right) \\
& +m_{\mathrm{T}}\left(\theta_{u}\left(t_{n}\right)\right) \cdot T_{\mathrm{z}, u}\left(t_{n}\right)+q_{1 m}^{2} I_{u, 1}^{k}\left(t_{n}\right) \\
& +\Delta \tilde{\rho}_{\mathrm{MP}, u, m}^{k}+\eta_{u, m}^{k}\left(t_{n}\right) .
\end{aligned}
$$

The reduced state vector includes the receiver position, the combined receiver clock/ reference ambiguity, the tropospheric zenith delay, the slant ionospheric delays, the differential integer ambiguities and the combined pseudorange multipath/ 
reference ambiguity parameters. It can be obtained from the full state vector of Eq. (5) by a linear transformation:

$$
\boldsymbol{x}_{\mathrm{red}, n}:=\left(\begin{array}{c}
\vec{x}_{u}\left(t_{n}\right) \\
c \delta \tilde{\boldsymbol{\tau}}_{u}\left(t_{n}\right) \\
T_{\mathrm{z}, u}\left(t_{n}\right) \\
\boldsymbol{I}_{u, 1}\left(t_{n}\right) \\
\Delta \boldsymbol{N}_{u} \\
\Delta \tilde{\boldsymbol{\rho}}_{\mathrm{MP}, u}\left(t_{n}\right)
\end{array}\right)=\boldsymbol{T} \boldsymbol{x}_{n}
$$

with the implicitly defined transformation matrix $\boldsymbol{T}$ and the following sub-state vectors:

$$
\begin{aligned}
c \delta \tilde{\boldsymbol{\tau}}_{u} & =\left(c \delta \tilde{\tau}_{u, 1}, \ldots, c \delta \tilde{\tau}_{u, M}\right)^{\mathrm{T}} \\
\Delta \boldsymbol{N}_{u} & =\left(\Delta \boldsymbol{N}_{u, 1}^{\mathrm{T}}, \ldots, \Delta \boldsymbol{N}_{u, M}^{\mathrm{T}}\right)^{\mathrm{T}} \\
\Delta \boldsymbol{N}_{u, m} & =\left(\Delta N_{u, m}^{1}, \ldots, \Delta N_{u, m}^{K}\right)^{\mathrm{T}} \\
\Delta \tilde{\boldsymbol{\rho}}_{\mathrm{MP}, u} & =\left(\Delta \tilde{\boldsymbol{\rho}}_{\mathrm{MP}, u, 1}^{\mathrm{T}}, \ldots, \Delta \tilde{\boldsymbol{\rho}}_{\mathrm{MP}, u, M}^{\mathrm{T}}\right)^{\mathrm{T}} \\
\Delta \tilde{\boldsymbol{\rho}}_{\mathrm{MP}, u, m} & =\left(\Delta \tilde{\rho}_{\mathrm{MP}, u, m}^{1}, \ldots, \Delta \tilde{\rho}_{\mathrm{MP}, u, m}^{K}\right)^{\mathrm{T}} .
\end{aligned}
$$

\section{PPP WITH KALMAN FILTER}

The PPP solution is determined with a standard linear Kalman filter. In this section, we briefly describe the state space model and the initialization of the state parameters.

\section{A. State space model}

The dynamics of the reduced state vector are described by a linear model:

$$
\boldsymbol{x}_{\mathrm{red}, n}=\boldsymbol{\Phi}_{n} \boldsymbol{x}_{\mathrm{red}, n-1}+\boldsymbol{\eta}_{x_{\mathrm{red}, n}},
$$

with the state transition matrix $\boldsymbol{\Phi}_{n}$, the reduced state vector $\boldsymbol{x}_{\text {red, } n-1}$ of the previous epoch and the process noise $\boldsymbol{\eta}_{x_{\mathrm{red}, n}}$. The state transition matrix is an identity matrix $\boldsymbol{I}$ for a static PPP solution, i.e. $\Phi_{n}=\boldsymbol{I}^{n_{x} \times n_{x}}$ with $n_{x}=5+K+2 M K$. For a kinematic PPP solution, the state vector typically also includes the velocity and in case of high dynamics even the acceleration as state parameters. In this case, the state transition matrix also includes off-diagonal elements to consider the integration of velocity/ acceleration states. However, we limit our simulation results to a static PPP solution in this paper and, thus, do not estimate velocity and acceleration as states.

\section{B. Initialization}

We initialize the state vector by least-squares estimation using the pre-corrected pseudorange and carrier phase measurements of a single epoch. The pseudorange multipath errors can not be estimated with single-epoch measurements. Thus, we determined the full-state vector of Eq. (5) except of pseudorange multipath parameters by minimizing the sum of squared measurement residuals:

$$
\left.\begin{array}{c}
\min _{\vec{x}_{u}\left(t_{1}\right)} \\
c \delta \tau_{u}\left(t_{1}\right) \\
T_{\mathrm{z}, u}\left(t_{1}\right) \\
\boldsymbol{I}_{u, 1}\left(t_{1}\right) \\
\Delta \boldsymbol{N}_{u}
\end{array}\right)\left\|\left(\begin{array}{c}
\tilde{\boldsymbol{\rho}}_{u, 1}\left(t_{1}\right) \\
\vdots \\
\tilde{\boldsymbol{\rho}}_{u, M}\left(t_{1}\right) \\
\lambda_{1} \tilde{\boldsymbol{\varphi}}_{u, 1}\left(t_{1}\right) \\
\vdots \\
\lambda_{M} \tilde{\boldsymbol{\varphi}}_{u, M}\left(t_{1}\right)
\end{array}\right)-\boldsymbol{H}\left(\begin{array}{c}
\vec{x}_{u}\left(t_{1}\right) \\
c \delta \tau_{u}\left(t_{1}\right) \\
T_{\mathrm{z}, u}\left(t_{1}\right) \\
\boldsymbol{I}_{u, 1}\left(t_{1}\right) \\
\Delta \boldsymbol{N}_{u}
\end{array}\right)\right\|^{2},
$$

with

$$
\begin{aligned}
\tilde{\boldsymbol{\rho}}_{u, m}\left(t_{1}\right) & =\left(\tilde{\rho}_{u, m}^{1}\left(t_{1}\right), \ldots, \tilde{\rho}_{u, m}^{K}\left(t_{1}\right)\right)^{\mathrm{T}} \\
\lambda_{m} \tilde{\boldsymbol{\varphi}}_{u, m}\left(t_{1}\right) & =\left(\lambda_{m} \tilde{\varphi}_{u, m}^{1}\left(t_{1}\right), \ldots, \lambda_{m} \varphi_{u, m}^{K}\left(t_{1}\right)\right)^{\mathrm{T}}
\end{aligned}
$$

The pseudorange multipath states of Eq. (5) are initialized with zeros, and its order of magnitude is considered in the state covariance matrix. After this initialization of the full state vector, we apply the linear transformation $T$ according to Eq. (10) to obtain an estimate of the reduced state parameters. The covariance matrix of $\hat{x}_{\mathrm{red}, n}$ follows from $\boldsymbol{\Sigma}_{\hat{x}_{\mathrm{red}, n}}=\boldsymbol{T} \boldsymbol{\Sigma}_{\hat{x}_{n}} \boldsymbol{T}^{\mathrm{T}}$.

\section{Simulation RESUlts}

In this section, we analyze the performance of PPP with Kepler. The first subsection describes the simulation parameter. Subsequently, we show the achievable accuracy and convergence behaviour for the absolute receiver position and clock offset, tropospheric zenith and ionospheric slant delays, carrier phase ambiguities and pseudorange multipath errors.

\section{A. Description of simulation parameters}

In this section, we describe our simulation scenario. Tab. I includes our receiver/ satellite geometry, and Tab. II and Tab. III show the assumed measurement and process noise statistics.

TABLE I

RECEIVER/ SATELLITE GEOMETRY

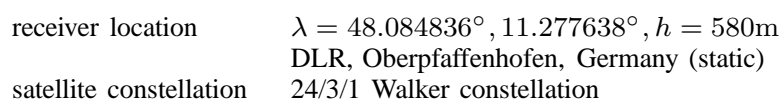

TABLE II

MEASUREMENT NOISE STATISTICS - STANDARD DEVIATIONS

$\begin{array}{lrr}\text { Frequency } & \text { code noise } & \text { phase noise } \\ \text { E1 } & 30 \mathrm{~cm} & 3 \mathrm{~mm} \\ \text { E5 } & 2 \mathrm{~cm} & 3 \mathrm{~mm} \\ \text { E6 } & 2 \mathrm{~cm} & 3 \mathrm{~mm}\end{array}$

TABLE III

PROCESS NOISE STATISTICS - STANDARD DEVIATIONS

Position

Receiver clock offset

Tropospheric zenith delay

Ionospheric zenith delays

Pseudorange multipath errors

$$
\begin{array}{r}
0 \mathrm{~m} \\
10 \mathrm{~m} / \mathrm{s} \\
2 \mathrm{~mm} / \mathrm{min} \\
10 \mathrm{~mm} / \mathrm{min} \\
1 \mathrm{~cm} / \mathrm{s}
\end{array}
$$

The pseudorange multipath errors of the first epoch were simulated as random values (statistically independent between satellites) from a Gaussian distribution with $\sigma=1 \mathrm{~m}$. 


\section{B. Description of simulation results}

Fig. 1 shows the error and standard deviation of the estimated $\mathrm{X}, \mathrm{Y}$ and Z-components of the absolute receiver position. A strong, unbiased convergence is observed. The errors of the absolute position drop below $20 \mathrm{~cm}$ within less than 5 minutes. This is much faster than today's GPS-based L1/L2 PPP solutions, which typically need more than 30 minutes to convergence.

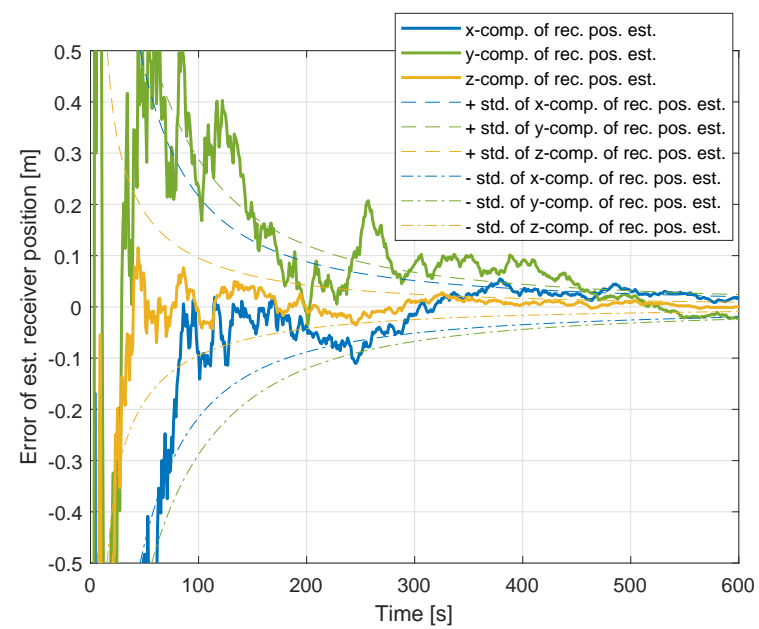

Fig. 1. Accuracy of absolute receiver position estimate

Fig. 2 shows the convergence behaviour of the receiver clock offset estimation. The convergence is much slower than for the receiver position due to the significantly larger process noise. The clock offset estimates are slightly biased due to the weak separability of some states, i.e. errors of other states are mapped into the clock offset.

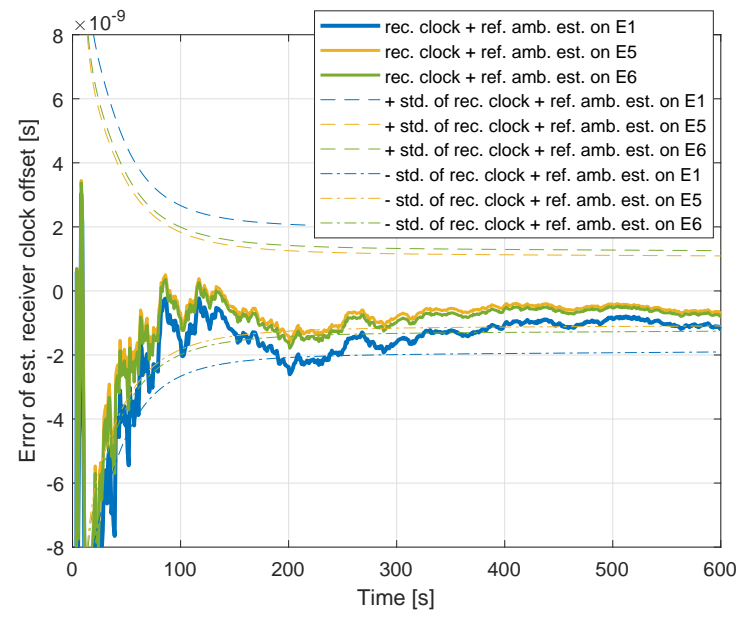

Fig. 2. Accuracy of receiver clock offset estimates

Fig. 3 shows the accuracy of the tropospheric zenith delay estimation. The standard deviation drops below $2 \mathrm{~cm}$ within 3 minutes and the estimates are unbiased.

The convergence of the ionospheric zenith delay estimation is shown in Fig. 4. The estimation of an individual zenith

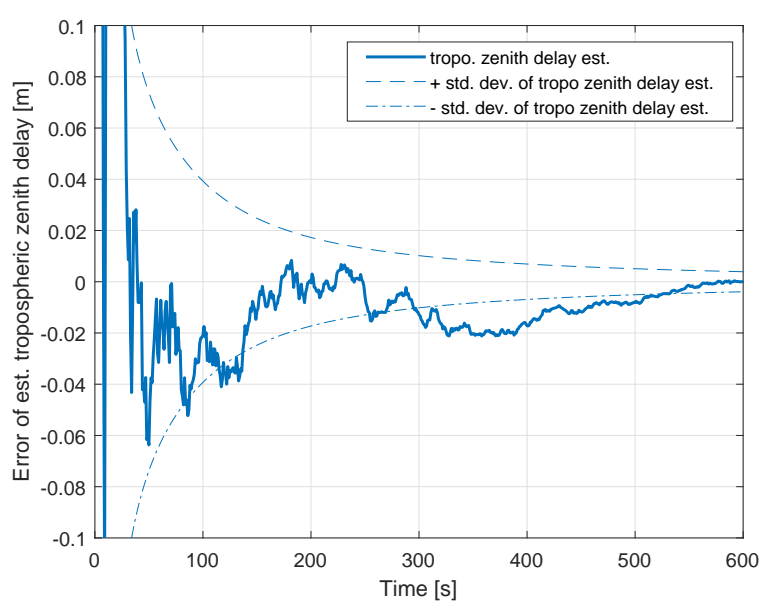

Fig. 3. Accuracy of tropospheric zenith delay estimates

delay for each satellite and the much larger process noise compared to the tropospheric zenith delay result in a slower convergence and higher errors of the ionospheric zenith delay estimation. However, these errors do not affect the absolute receiver position, which is determined much more accurately.

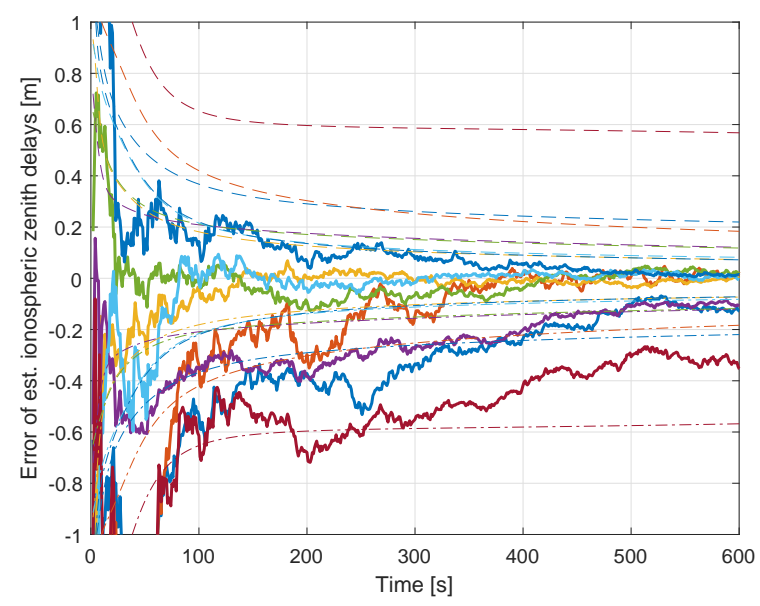

Fig. 4. Accuracy of ionospheric zenith delay estimates on E1. Each color refers to a different Galileo satellite.

Fig. 5 shows the accuracy of the float ambiguity estimates. The errors of different satellites and frequencies are significantly correlated, which could be exploited by an integer decorrelation and a subsequent ambiguity fixing [4].

Fig. 6 shows the simulated pseudorange multipath errors and Fig. 7 provides the accuracy of their estimates. The errors are again significantly correlated.

Fig. 8 and 9 show the residuals of the phase and pseudorange measurements of all satellites and frequencies. All residuals are unbiased and the magnitude is in the order of the measurement noise, which confirms the consistency of our state estimation and measurement models. 


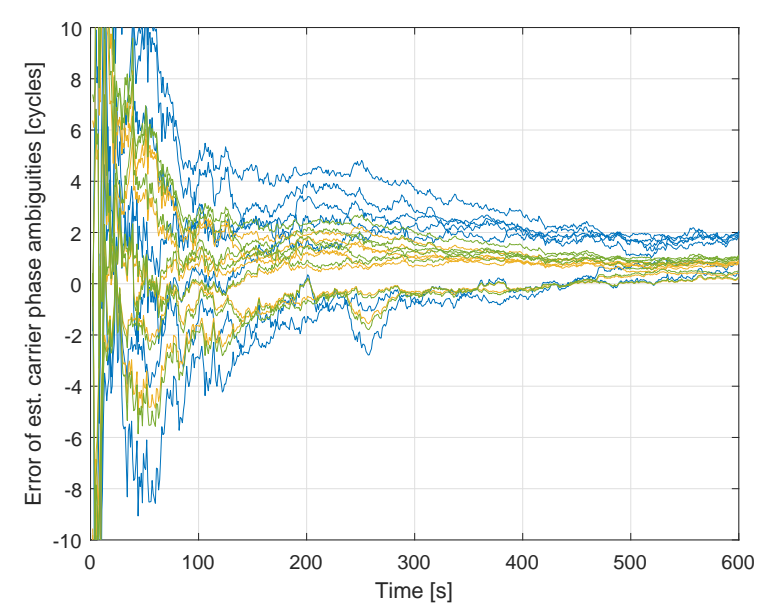

Fig. 5. Accuracy of carrier phase ambiguity estimates. Each color refers to a different frequency (E1, E5, E6). Different curves of the same color refer to different satellites.

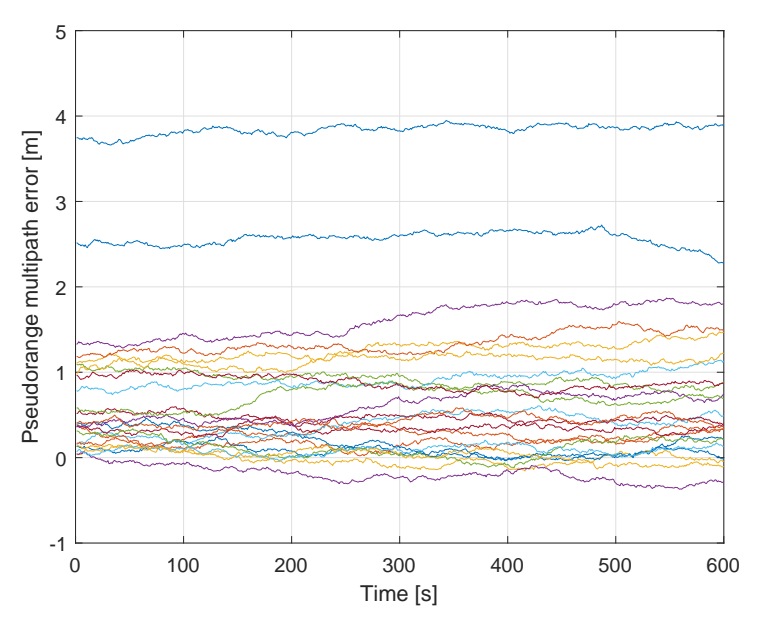

Fig. 6. Simulated pseudorange multipath errors for a static receiver. Different curves refer to different satellites and frequencies.

\section{CONCLUSION}

The broad use of PPP requires a faster convergence than today's L1/ L2-based GPS solutions. This paper has provided a PPP solution that exploits the highly accurate position, clock and bias estimates of next-generation GNSS Kepler and the low noise level of the wideband signals on E1, E5 and E6. Thereby, the convergence time was reduced from 30 minutes to less than 5 minutes without any prior information.

\section{REFERENCES}

[1] J. Zumberge, M. Heflin, D. Jefferson, M. Watkins, and F. Webb: "Precise point positioning for the efficient and robust analysis of GPS data from large network", J. of Geophysical Res., vol. 102, pp. 5005-5017, 1997.

[2] C. Günther: "Kepler - Satellite Navigation without Clocks and Ground Infrastr.", Proc. of ION GNSS+, pp. 849 - 856, Miami, USA, Sep 2018.

[3] J. Kouba: "A guide to using intern. GNSS service (IGS) products", 2009.

[4] P. Teunissen: "The Least-Squares Ambiguity Decorrelation Adjustment - A method for fast GPS ambiguity fixing", J. of Geodesy, vol. 70, pp. $65-82,1995$.

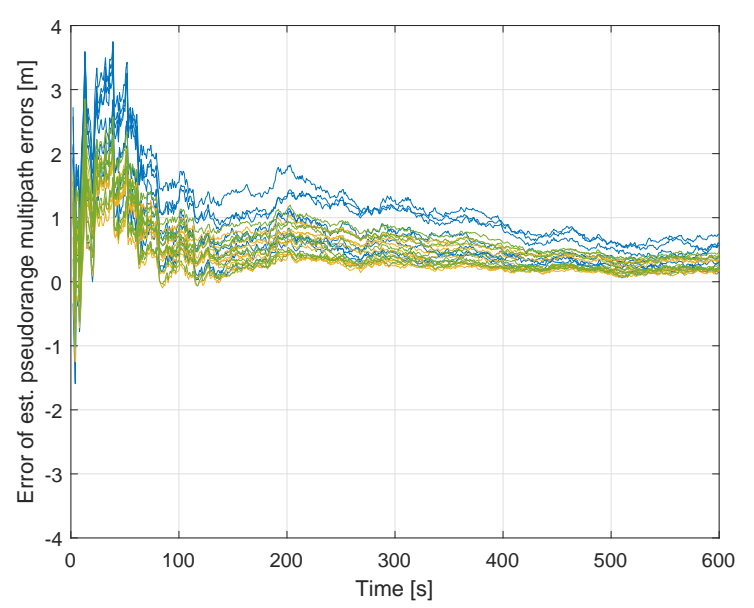

Fig. 7. Accuracy of pseudorange multipath estimates. Each color refers to a different frequency. Different curves of one color refer to different satellites.

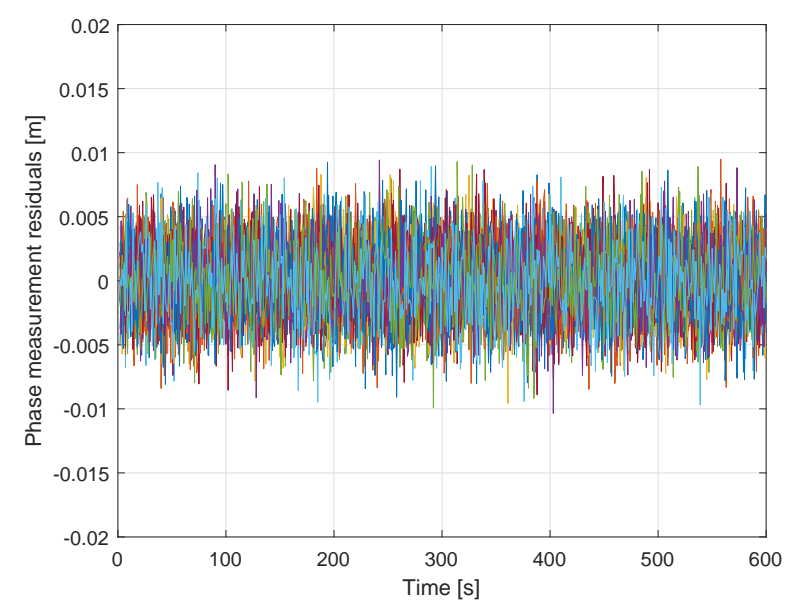

Fig. 8. Phase measurement residuals of all satellites and frequencies.

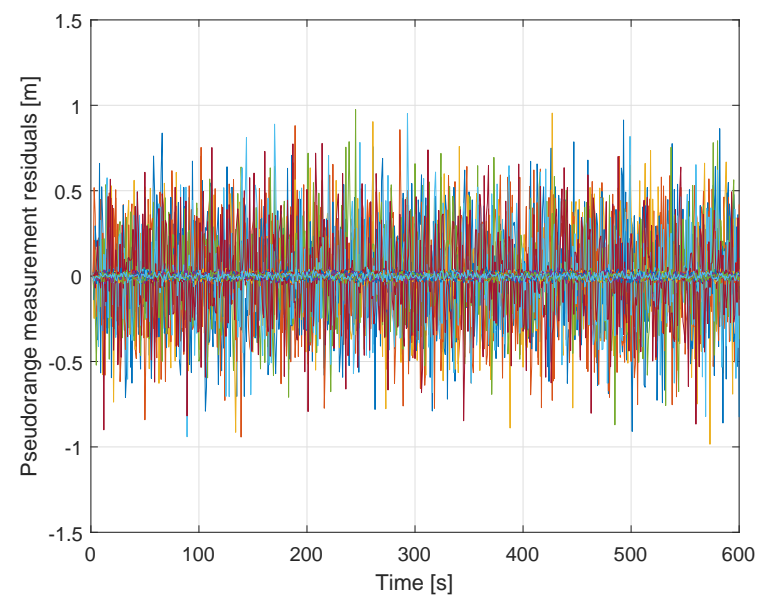

Fig. 9. Pseudorange measurement residuals of all satellites and frequencies. 